Proceedings of the Edinburgh Mathematical Society (2007) 50, 637-652 (C)

DOI:10.1017/S0013091505001124 Printed in the United Kingdom

\title{
ON OPERATORS CAUCHY DUAL TO 2-HYPEREXPANSIVE OPERATORS
}

\author{
SAMEER CHAVAN \\ Department of Mathematics, University of Pune, Pune 411007, India \\ (sameer_chavan95@yahoo.co.in)
}

(Received 3 August 2005)

\begin{abstract}
The operator Cauchy dual to a 2-hyperexpansive operator $T$, given by $T^{\prime} \equiv T\left(T^{*} T\right)^{-1}$, turns out to be a hyponormal contraction. This simple observation leads to a structure theorem for the $C^{*}$-algebra generated by a 2-hyperexpansion, and a version of the Berger-Shaw theorem for 2-hyperexpansions.

As an application of the hyperexpansivity version of the Berger-Shaw theorem, we show that every analytic 2-hyperexpansive operator with finite-dimensional cokernel is unitarily equivalent to a compact perturbation of a unilateral shift.
\end{abstract}

Keywords: hyponormal contraction; 2-hyperexpansive operator; Cauchy dual

2000 Mathematics subject classification: Primary 47B20

\section{Preliminaries}

Completely hyperexpansive operators were introduced in [4] and studied extensively by Athavale and co-workers (see $[\mathbf{5 - 8 , 2 5}]$ ) as well as by Jabloński and Stochel (see $[\mathbf{1 4}-\mathbf{1 8}]$ ). The class of completely hyperexpansive operators is closely related to the theory of negative definite functions on abelian semigroups, and it is in some sense antithetical to the class of subnormal contractions. In the present paper, we deal with the superclass of complete hyperexpansions of 2-hyperexpansions and exploit the theory of hyponormals to reveal some interesting facts about 2-hyperexpansions. The notion of the Cauchy dual operator introduced by Shimorin [24] enables us to think of the theory of 2-hyperexpansions as an antithesis of the theory of hyponormal contractions. For all the elementary results pertaining to hyponormal operators, the reader is referred to $[\mathbf{1 0}, \mathbf{2 0}]$.

Unless stated otherwise, all the Hilbert spaces occurring below are complex, infinite dimensional and separable, and for any such Hilbert space $\mathcal{H}, \mathcal{B}(\mathcal{H})$ denotes the algebra of bounded linear operators on $\mathcal{H}$. In what follows, for any bounded linear operator $T$ on $\mathcal{H}$, we denote the spectrum, the point spectrum, the approximate point spectrum and the essential spectrum of $T$ by $\sigma(T), \sigma_{\mathrm{p}}(T), \sigma_{\mathrm{ap}}(T)$ and $\sigma_{\mathrm{e}}(T)$, respectively. The symbols $\operatorname{null}(T)$ and $\operatorname{ran}(T)$ will stand for the null-space and the range-space of $T$, respectively. If $W$ is a subset of $\mathcal{H}$, then linspan $W$ will stand for the smallest linear manifold generated 
by $W$. By $\vee\{w: w \in W\}$, we mean the smallest closed linear manifold generated by $W$. If $\left\{e_{n}\right\}_{n \geqslant 0}$ is an orthonormal basis for a Hilbert space $\mathcal{H}$, then a weighted shift operator $T$ in $\mathcal{B}(\mathcal{H})$ with the weight sequence $\left\{\alpha_{n}\right\}_{n \geqslant 0}$ is defined through the relations $T e_{n}=\alpha_{n} e_{n+1}$ for every $n \geqslant 0$. We will assume that $\alpha_{n}$ are positive for all $n \geqslant 0$. We will use the notation $T:\left\{\alpha_{n}\right\}_{n \geqslant 0}$ to indicate a weighted shift. An excellent reference for the basic properties of weighted shifts is $[\mathbf{2 3}]$.

Recall that $S$ in $\mathcal{B}(\mathcal{H})$ is subnormal if there exist a Hilbert space $\mathcal{K}$ containing $\mathcal{H}$ and a normal operator $N$ in $\mathcal{B}(\mathcal{K})$ such that $N h=S h$ for every $h \in \mathcal{H}$. Agler proved in [1] that $S$ in $\mathcal{B}(\mathcal{H})$ is a subnormal contraction (that is, $S$ is subnormal and $\|S\| \leqslant 1$ ) if and only if

$$
B_{n}(S) \equiv \sum_{0 \leqslant p \leqslant n}(-1)^{p}\left(\begin{array}{l}
n \\
p
\end{array}\right) S^{* p} S^{p} \geqslant 0 \quad \text { for all integers } n \geqslant 0 .
$$

If, for a positive integer $m$, an operator $S$ in $\mathcal{B}(\mathcal{H})$ satisfies the inequalities in (1.1) for $1 \leqslant n \leqslant m$, then $S$ is said to be $m$-hypercontractive.

The operator Cauchy dual to a 2-hyperexpansion, which is a hyponormal contraction, turns out to be the key observation in our analysis of the present paper. We illustrate how this observation can be used to unravel some interesting facts about 2-hyperexpansions such as a rich supply of non-zero $*$-homomorphisms on the $C^{*}$-algebra generated by a pure 2-hyperexpansion and a realization of an analytic finitely multicyclic 2-hyperexpansion as a compact perturbation of a unilateral shift. This paper is an attempt to develop a theory of 2-hyperexpansive operators parallel to the theory of hyponormals.

\section{The Cauchy dual operator}

Definition 2.1. Let $T$ be in $\mathcal{B}(\mathcal{H})$. Then $T$ is said to be completely hyperexpansive if

$$
B_{n}(T) \equiv \sum_{0 \leqslant p \leqslant n}(-1)^{p}\left(\begin{array}{l}
n \\
p
\end{array}\right) T^{* p} T^{p} \leqslant 0 \quad \text { for all integers } n \geqslant 1 .
$$

Let $m$ be a positive integer. If $T$ satisfies the inequalities in (2.1) for $1 \leqslant n \leqslant m$, then $T$ is said to be $m$-hyperexpansive. If $T$ satisfies $B_{m}(T)=0$, then $T$ is said to be $m$-isometric. $T$ is said to be expansive (respectively isometric) if $T$ is 1-expansive (respectively 1-isometric).

Remark 2.2. Note that an expansive operator is left-invertible.

Definition 2.3. Let $T$ in $\mathcal{B}(\mathcal{H})$ be left-invertible. Then the operator $T^{\prime} \equiv T\left(T^{*} T\right)^{-1}$ is said to be the operator Cauchy dual to $T$.

Remark 2.4. Note that, for any left-invertible $T$ in $\mathcal{B}(\mathcal{H})$, one has $T^{*} T^{\prime}=\left(T^{*} T\right)^{-1}$. Hence, the Cauchy dual operator $T^{\prime}$ is left-invertible, and the operator Cauchy dual to $T^{\prime}$ is $T$, that is, $\left(T^{\prime}\right)^{\prime}=T$. 
The following example justifies the terminology used for $T^{\prime}[\mathbf{2 4}, \S 1]$.

Example 2.5. Let $T:\left\{\alpha_{n}\right\}_{n \geqslant 0}$ be a weighted shift operator. Note that $T:\left\{\alpha_{n}\right\}_{n \geqslant 0}$ is left-invertible if and only if the infimum $\inf _{n \geqslant 0} \alpha_{n}$ of the weight sequence $\left\{\alpha_{n}\right\}_{n \geqslant 0}$ is positive. In the case when $\inf _{n \geqslant 0} \alpha_{n}>0$, it is easy to see that the operator Cauchy dual to the weighted shift $T:\left\{\alpha_{n}\right\}_{n \geqslant 0}$ is given by the weighted shift $T^{\prime}:\left\{1 / \alpha_{n}\right\}_{n \geqslant 0}$.

Let $\alpha$ be a non-negative real number and let $\Gamma$ be the standard gamma function. Let $\mathcal{H}_{\alpha}$ denote the Hilbert space of analytic functions on the unit disc $\mathbb{D}$ with the inner product

$$
\langle f, g\rangle_{\alpha}=\sum_{n=0}^{\infty} \frac{\Gamma(\alpha+1+n)}{\Gamma(\alpha+1) n !} a_{n} \bar{b}_{n},
$$

where $f=\sum_{n=0}^{\infty} a_{n}(\cdot)^{n}$ and $g=\sum_{n=0}^{\infty} b_{n}(\cdot)^{n}$. Then the Hilbert space Cauchy dual to $\mathcal{H}_{\alpha}$ is the Hilbert space $\mathcal{H}_{-\alpha}$ of analytic functions on the unit disc $\mathbb{D}$ with the inner product

$$
\langle f, g\rangle_{-\alpha}=\sum_{n=0}^{\infty} \frac{\Gamma(\alpha+1) n !}{\Gamma(\alpha+1+n)} a_{n} \bar{b}_{n} ;
$$

the dual pairing between $\mathcal{H}_{\alpha}$ and $\mathcal{H}_{-\alpha}$ is given by

$$
(f, g)=\sum_{n=0}^{\infty} a_{n} \bar{b}_{n},
$$

where $f \in \mathcal{H}_{\alpha}, g \in \mathcal{H}_{-\alpha}$. Let $M_{\text {. }}^{\alpha}$ be the operator of multiplication by the coordinate function '.' on $\mathcal{H}_{\alpha}$. For $0 \leqslant \alpha \leqslant 1$, the operator $M^{\alpha}$. on $\mathcal{H}_{\alpha}$ is completely hyperexpansive, while the Cauchy dual operator $\left(M_{*}^{\alpha}\right)^{\prime}=M^{-\alpha}$ on $\mathcal{H}_{-\alpha}$ is a subnormal contraction [25]. For $-1<\beta \leqslant 0$, one can easily check that the operator $M^{\beta}$ on $\mathcal{H}_{\beta}$ is completely hyperexpansive; in fact,

$$
\left\langle B_{\mathrm{p}}\left(M^{\beta}\right) e_{m}, e_{m}\right\rangle=\frac{\beta(1+\beta) \cdots(p-1+\beta)}{(m+1+\beta) \cdots(m+p+\beta)},
$$

where

$$
e_{m}=\frac{(\cdot)^{m}}{\left\|(\cdot)^{m}\right\|_{\beta}}
$$

for $m \geqslant 0$, being the coordinate function $\cdot(z)=z, z \in \mathbb{D}$, while the Cauchy dual operator $\left(M^{\beta}\right)^{\prime}=M^{-\beta}$ on $\mathcal{H}_{-\beta}$ is a subnormal contraction (see Proposition 2.6, below).

The association $T \mapsto T^{\prime}$ is studied by Athavale [4] in the context of weighted shift operators, while the notion of Cauchy dual operator (Definition 2.3) is introduced by Shimorin in [24]. Note that the operator Cauchy dual to an expansion is a contraction. For some special expansive operators more can be said; in [4], it is proved that the operator Cauchy dual to a completely hyperexpansive weighted shift is a contractive subnormal weighted shift. Indeed, we have the following. 
Proposition 2.6 (Athavale [4, Proposition 6]). Let $T:\left\{\alpha_{n}\right\}_{n \geqslant 0}$ be a weighted shift. Set $\beta_{0}=1$ and $\beta_{n}=\alpha_{0}^{2} \ldots \alpha_{n-1}^{2}, n \geqslant 1$. Then $T$ is completely hyperexpansive if and only if

$$
S_{t}:\left\{\sqrt{\frac{t\left(\beta_{n}-1\right)+1}{t\left(\beta_{n+1}-1\right)+1}}\right\}_{n \geqslant 0}
$$

is a contractive subnormal weighted shift for every $t>0$.

One more motivation for studying the operator Cauchy dual to a left-invertible operator $T$ lies in the fact that $T^{\prime}=T\left(T^{*} T\right)^{-1}$ forms a complete unitary invariant for $T$. In view of this remark and Proposition 2.6, it is interesting to know more about operators Cauchy dual to non-isometric left-invertible operators. One such result is established in [24], namely, the operator Cauchy dual to a 2-hyperexpansion is 2-hypercontractive. Indeed, it is observed in [24] that, for a 2-hyperexpansive $T$ in $\mathcal{B}(\mathcal{H})$, the Cauchy dual operator $T^{\prime}=T\left(T^{*} T\right)^{-1}$ satisfies

$$
\left\|T^{\prime} x+T^{\prime} y\right\|^{2} \leqslant 2\left(\|x\|^{2}+\left\|T^{\prime 2} y\right\|^{2}\right), \quad x, y \in \mathcal{H}
$$

(see the remark following [24, Corollary 3.7]).

The following proposition sheds light on the spectral behaviour of 2-hyperexpansions. The proof rests on the simple observation that the operator Cauchy dual to an expansion is a contraction.

Proposition 2.7. Let $T$ in $\mathcal{B}(\mathcal{H})$ be a 2-hyperexpansion. The following are then true.

(1) For every $\mu$ in the point spectrum $\sigma_{\mathrm{p}}(T)$ of $T$, the null-space null $(T-\mu I)$ of $T-\mu I$ reduces $T$ and $\left.T\right|_{\operatorname{null}(T-\mu I)}$ is unitary.

(2) If $f_{1}$ and $f_{2}$ are eigenvectors corresponding to distinct eigenvalues of $T$, then $\left\langle f_{1}, f_{2}\right\rangle=0$.

If in addition $T$ is pure (that is, $T$ has no proper normal direct summand), then

(3) $\sigma_{\mathrm{p}}(T)=\emptyset$ and

(4) the Cayley transform $\mathcal{C}: \operatorname{ran}(T+I) \rightarrow \operatorname{ran}(T-I)$ given by

$$
\mathcal{C}(T+I) h=(T-I) h, \quad h \in \mathcal{H},
$$

is accretive (that is, $\langle\mathcal{C} x, x\rangle+\langle x, \mathcal{C} x\rangle \geqslant 0$ for any $x \in \mathcal{H}$ ).

Proof. Suppose that $T$ in $\mathcal{B}(\mathcal{H})$ is 2-hyperexpansive. The point spectrum $\sigma_{\mathrm{p}}(T)$ of $T$ is contained in the unit circle $\partial \mathbb{D} \equiv\{z \in \mathbb{C}:|z|=1\}$ of the complex plane $\mathbb{C}[\mathbf{2 5}$, Remark 3.4]. Let $\mu \in \mathbb{C}$ be such that $|\mu|=1$ and let $h \in \mathcal{H}$ be such that $T h=\mu h$. Thus, $T^{*} T h=$ $\mu T^{*} h, h=\mu T^{* *} h$. Since $\mu T^{* *}$ is a contraction, by [26, Proposition 3.1, Chapter 1], one has $h=\bar{\mu} T^{\prime} h$. Using $T^{*} T^{\prime}=I$, one has $T^{*} h=\bar{\mu} h$. Consequently, $\operatorname{null}(T-\mu I)$ reduces $T$ and $\left.T\right|_{\text {null }}(T-\mu I)$ is normal for every $\mu \in \sigma_{\mathrm{p}}(T)$. Since the restriction of a 2-hyperexpansion to an invariant subspace is a 2 -hyperexpansion, $\left.T\right|_{\text {null }}(T-\mu I)$ is a normal 2-hyperexpansion. Hence, $\left.T\right|_{\text {null }}(T-\mu I)$ is an invertible 2-hyperexpansion and is unitary [25, Remark 3.4]. This completes the proof of (1). Part (3) is now obvious. 
Next let $f_{1}, f_{2} \in \mathcal{H}$ be such that $T f_{1}=\mu_{1} f_{1}$ and $T f_{2}=\mu_{2} f_{2}$ for some $\mu_{1}, \mu_{2} \in \mathbb{C}$. An examination of the preceding paragraph reveals that $T^{*} f_{2}=\bar{\mu}_{2} f_{2}$. Then

$$
\mu_{1}\left\langle f_{1}, f_{2}\right\rangle=\left\langle T f_{1}, f_{2}\right\rangle=\left\langle f_{1}, T^{*} f_{2}\right\rangle=\mu_{2}\left\langle f_{1}, f_{2}\right\rangle .
$$

Hence, $\left\langle f_{1}, f_{2}\right\rangle=0$ if $\mu_{1} \neq \mu_{2}$. This establishes part (2).

To prove (4), assume in addition that $T$ is pure. Since $T$ is pure, by part (1), $\operatorname{null}(T+I)$ is trivial. Thus, the Cayley transform $\mathcal{C}$ is well defined. Also, the real part of $\langle\mathcal{C}(T+I) h,(T+I) h\rangle$ is non-negative: if $\operatorname{Re} z$ denotes the real part of a complex number $z$, then

$$
\begin{aligned}
\langle\mathcal{C}(T+I) h,(T+I) h\rangle+\langle(T+I) h, \mathcal{C}(T+I) h\rangle & =2 \operatorname{Re}\langle(T-I) h,(T+I) h\rangle \\
& =2\left(\|T h\|^{2}-\|h\|^{2}\right) \\
& \geqslant 0
\end{aligned}
$$

for every $h \in \mathcal{H}$.

Remark 2.8. The proof of part (1) above shows that if an expansive operator $T \in$ $\mathcal{B}(\mathcal{H})$ fixes a point $h$ in $\mathcal{H}$, then so does $T^{*}$. Also, from the proof of part (4) above, it is clear that the Cayley transform $\mathcal{C}$ of any pure expansive $T$ is accretive. If in addition $T$ is invertible, then it can be seen that $\mathcal{C}$ is $m$-accretive (that is, $\mathcal{C}$ has no proper accretive extension in $\mathcal{H})[\mathbf{2 6}]$.

An operator $S$ in $\mathcal{B}(\mathcal{H})$ is said to be hyponormal if the self-commutator $\left[S^{*}, S\right]=$ $S^{*} S-S S^{*}$ of $S$ is a positive operator. Recall that $S$ is hyponormal if and only if there exists a contraction $C$ in $\mathcal{B}(\mathcal{H})$ such that $S^{*}=C S[\mathbf{2 0}]$.

Theorem 2.9. Let $T$ in $\mathcal{B}(\mathcal{H})$ be 2-hyperexpansive and let $T^{\prime}$ be the operator Cauchy dual to $T$. There then exists a contraction $C$ similar to an isometry such that $T^{\prime *}=C^{*} T^{\prime}$. Thus, the operator Cauchy dual to a 2-hyperexpansion is a hyponormal contraction.

Proof. Let $T$ be a 2-hyperexpansion and let $T^{\prime}$ be the operator Cauchy dual to $T$. Since

$$
\left(I-T^{*} T\right)^{2}+T^{* 2} T^{2}-\left(T^{*} T\right)^{2}=I-2 T^{*} T+T^{* 2} T^{2},
$$

and $I-2 T^{*} T+T^{* 2} T^{2} \leqslant 0$, one has $T^{* 2} T^{2} \leqslant\left(T^{*} T\right)^{2}$. Thus, $\left\|T^{2} x\right\| \leqslant\left\|T^{*} T x\right\|, x \in \mathcal{H}$. Since $T=T^{\prime}\left(T^{*} T^{\prime}\right)^{-1}$ and $T^{*} T=\left(T^{* *} T^{\prime}\right)^{-1}$, one has

$$
\left\|T T^{\prime}\left(T^{* *} T^{\prime}\right)^{-1} x\right\| \leqslant\left\|\left(T^{* *} T^{\prime}\right)^{-1} x\right\|, \quad x \in \mathcal{H} .
$$

Thus,

$$
\left\|T T^{\prime} y\right\| \leqslant\|y\|, \quad y \in \mathcal{H}
$$

Hence, $T T^{\prime}$ is a contraction. Since $\left(T T^{\prime}\right)^{*} T^{\prime}=T^{* *}$, the desired $C$ is $T T^{\prime}$ provided that $T T^{\prime}$ is similar to an isometry. To prove that $T T^{\prime}$ is similar to an isometry, by $[\mathbf{1 9}$, 
Corollary 1.16] (and the discussion following it), it suffices to show that there exist some positive constants $m, M$ such that

$$
m\|x\| \leqslant\left\|\left(T T^{\prime}\right)^{n} x\right\| \leqslant M\|x\|, \quad n \geqslant 0 .
$$

First we check that $T^{\prime} T$ is expansive. Because of the 2-hyperexpansivity, one has

$$
\|x\|\left\|T^{2} x\right\| \leqslant \frac{1}{2}\left(\|x\|^{2}+\left\|T^{2} x\right\|^{2}\right) \leqslant\|T x\|^{2}, \quad x \in \mathcal{H} .
$$

Thus,

$$
\|x\|\left\|T^{2} x\right\| \leqslant\|T x\|^{2}, \quad x \in \mathcal{H} .
$$

Using $T=T^{\prime}\left(T^{*} T^{\prime}\right)^{-1}$, one has

$$
\|x\|\left\|T T^{\prime}\left(T^{*} T^{\prime}\right)^{-1} x\right\| \leqslant\left\|T^{\prime}\left(T^{*} T^{\prime}\right)^{-1} x\right\|^{2}, \quad x \in \mathcal{H} .
$$

Thus,

$$
\left\|T^{* *} T^{\prime} y\right\|\left\|T T^{\prime} y\right\| \leqslant\left\|T^{\prime} y\right\|^{2}, \quad y \in \mathcal{H} .
$$

Since $T^{*} T^{\prime}=I$, we must have

$$
\left\|T^{\prime} y\right\|^{2}=\left\langle T^{\prime} y, T^{\prime} y\right\rangle=\left\langle T^{*} T^{\prime}\left(T^{\prime} y\right), T^{\prime} y\right\rangle=\left\langle T^{\prime 2} y, T T^{\prime} y\right\rangle \leqslant\left\|T^{\prime 2} y\right\|\left\|T T^{\prime} y\right\|, \quad y \in \mathcal{H} .
$$

By (2.3) and the previous inequality, one has

$$
\left\|T^{\prime *} T^{\prime} y\right\|\left\|T T^{\prime} y\right\| \leqslant\left\|T^{\prime} y\right\|^{2} \leqslant\left\|T^{\prime 2} y\right\|\left\|T T^{\prime} y\right\|, \quad y \in \mathcal{H} .
$$

Since $T T^{\prime}$ is injective, one has $\left\|T^{*} T^{\prime} y\right\| \leqslant\left\|T^{\prime 2} y\right\|$, and hence $\|y\| \leqslant\left\|T^{\prime 2}\left(T^{* *} T^{\prime}\right)^{-1} y\right\|$ for every $y \in \mathcal{H}$. Using $T=T^{\prime}\left(T^{* *} T^{\prime}\right)^{-1}$, one has $\|y\| \leqslant\left\|T^{\prime} T y\right\|, y \in \mathcal{H}$, that is $T^{\prime} T$ is expansive.

Now we check that (2.2) holds for some positive constants $m$ and $M$. Since $T T^{\prime}$ is a contraction, one can choose $M=1$. Also, since $T$ and $T^{\prime} T$ are expansive, one has

$$
\left\|\left(T T^{\prime}\right)^{n} x\right\|=\left\|T\left(T^{\prime} T\right)^{n-1} T^{\prime} x\right\| \geqslant\left\|\left(T^{\prime} T\right)^{n-1} T^{\prime} x\right\| \geqslant\left\|T^{\prime} x\right\|, \quad x \in \mathcal{H} .
$$

Since $T^{\prime}$ is bounded below, (2.2) follows.

Remark 2.10. In view of the proof of the preceding theorem, for any left-invertible $T$ in $\mathcal{B}(\mathcal{H})$, one has

$$
T^{*}\left[T^{*}, T\right] T \leqslant 0 \quad \Longrightarrow \quad\left[T^{* *}, T^{\prime}\right] \geqslant 0 .
$$

If $T$ is a pure left-invertible operator, then it can easily be seen that the Cauchy dual operator $T^{\prime}$ is pure. From the remark following Proposition 2.6, it follows readily that any complete set of unitary invariants for $T^{\prime}$ forms a complete set of unitary invariants for $T$. In view of these observations, Theorem 2.9 and [20, Proposition 3.8, Chapter II], the operator-valued function $C^{*}(\cdot) C(\cdot): \mathbb{C} \times \mathbb{C} \rightarrow \mathcal{B}(\overline{\operatorname{ran}(D)})$ given by

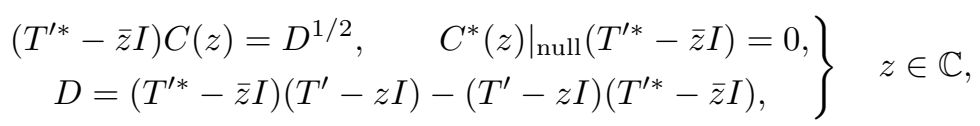

is a complete unitary invariant for any pure 2-hyperexpansive $T$. 
Question 2.11. Is the operator Cauchy dual to a completely hyperexpansive operator a subnormal contraction?

Corollary 2.12. If $S$ in $\mathcal{B}(\mathcal{H})$ satisfies $\|S x+y\|^{2} \leqslant 2\left(\|x\|^{2}+\|S y\|^{2}\right), x, y \in \mathcal{H}$, then $S$ is a hyponormal contraction.

Proof. Suppose that $S$ in $\mathcal{B}(\mathcal{H})$ satisfies $\|S x+y\|^{2} \leqslant 2\left(\|x\|^{2}+\|S y\|^{2}\right), x, y \in \mathcal{H}$. It is recorded in the proof of [24, Theorem 3.6] that the Cauchy dual operator $S^{\prime}$ is 2-hyperexpansive. Since $\left(S^{\prime}\right)^{\prime}=S$, one may appeal to Theorem 2.9.

\subsection{Applications}

In this subsection, we give several applications of Theorem 2.9 to the theory of hyperexpansive operators. These are an alternative proof of a result of Richter [22] on the wandering subspaces of analytic 2-hyperexpansions (Corollary 2.18), a structure theorem for the $C^{*}$-algebra generated by a 2-hyperexpansion (Proposition 2.16), and a version of the Berger-Shaw theorem for 2-hyperexpansions (Proposition 2.21). The arguments to follow rely heavily on the theory of hyponormal operators as expounded in $[\mathbf{1 0}, \mathbf{2 0}]$.

For $S \in \mathcal{B}(\mathcal{H})$, let $C^{*}(S)$ denote the $C^{*}$-algebra generated by $S$, that is, $C^{*}(S)$ is the norm closure in $\mathcal{B}(\mathcal{H})$ of the linear manifold of all non-commutative polynomials $p$ in $S$ and $S^{*}$ such that $p(0,0)=0$. We do not assume that the identity operator $I$ belongs to $C^{*}(S)$.

Lemma 2.13. Let $T$ in $\mathcal{B}(\mathcal{H})$ be left-invertible and let $T^{\prime}$ be the operator Cauchy dual to $T$. Then the identity operator $I$ belongs to $C^{*}(T)$ and $C^{*}\left(T^{\prime}\right)=C^{*}(T)$.

Proof. Let $T$ in $\mathcal{B}(\mathcal{H})$ be left-invertible and let $T^{\prime}$ be the operator Cauchy dual to $T$. In order to establish the equality $C^{*}\left(T^{\prime}\right)=C^{*}(T)$, in view of $\left(T^{\prime}\right)^{\prime}=T$ it suffices to check that $C^{*}\left(T^{\prime}\right) \subset C^{*}(T)$. Since $T^{*} T$ is an invertible positive operator, $\sigma\left(T^{*} T\right)$ is a compact subset of the open interval $(0, \infty)$. By a straightforward application of the Stone-Weiestrass theorem [11, Corollary 8.2, Chapter V] one has

$$
\text { the uniform closure of } \operatorname{linspan}\left\{(\cdot)^{n}: n \geqslant 1\right\}=C\left(\sigma\left(T^{*} T\right)\right) \text {, }
$$

the sup-normed Banach space of all continuous complex functions on $\sigma\left(T^{*} T\right)$, where the function $(\cdot)^{n}$ defined on $\sigma\left(T^{*} T\right)$ is given by $(\cdot)^{n}(t)=t^{n}, t \in \sigma\left(T^{*} T\right), n \in \mathbb{N}$. Thus, one can approximate the function

$$
\frac{1}{(\cdot)}: \sigma\left(T^{*} T\right) \rightarrow(0, \infty), \quad \frac{1}{(\cdot)}(t)=\frac{1}{t}, \quad t \in \sigma\left(T^{*} T\right),
$$

by functions in linspan $\left\{(\cdot)^{n}: n \geqslant 1\right\}$ in the sup-norm. Hence, by the spectral theorem one can approximate $\left(T^{*} T\right)^{-1}$ by operators in linspan $\left\{\left(T^{*} T\right)^{n}: n \geqslant 1\right\}$ in the operator norm. Therefore, $T^{\prime}$ belongs to $C^{*}(T)$ and we have the inclusion $C^{*}\left(T^{\prime}\right) \subset C^{*}(T)$. Since $T^{*} T$, $\left(T^{*} T\right)^{-1}$ are in $C^{*}(T)$, one has $I \in C^{*}(T)$. This completes the proof of the lemma.

The following lemma, which is crucial in our analysis, is of independent interest. 
Lemma 2.14. Let $T$ in $\mathcal{B}(\mathcal{H})$ be a 2-hyperexpansion and let $T^{\prime}$ be the operator Cauchy dual to $T$. Let $\mathbb{D}$ and $\partial \mathbb{D}$ denote the open unit disc $\{z \in \mathbb{C}:|z|<1\}$ and the unit circle $\{z \in \mathbb{C}:|z|=1\}$ in the complex plane $\mathbb{C}$, respectively. Then the following are true.

(i) $\sigma\left(T^{\prime}\right)=\sigma(T) \subset \overline{\mathbb{D}}$ and $\sigma_{\text {ap }}\left(T^{\prime}\right)=\sigma_{\text {ap }}(T) \subset \partial \mathbb{D}$.

(ii) $\sigma\left(T^{\prime}\right)=\overline{\mathbb{D}}$ and $\sigma_{\mathrm{ap}}\left(T^{\prime}\right)=\partial \mathbb{D}$, provided that $T$ is not invertible.

Proof. Let $T$ in $\mathcal{B}(\mathcal{H})$ be a 2-hyperexpansion and let $T^{\prime}$ be the operator Cauchy dual to $T$. If $T$ is invertible, then $T$ is unitary [25, Remark 3.4]. In this case, $T^{\prime}=T$ and (i) follows. So we assume that $T$ is not invertible. It is known that $\sigma(T)=\overline{\mathbb{D}}$ and $\sigma_{\text {ap }}(T)=$ $\partial \mathbb{D}$ hold for any non-invertible 2-hyperexpansion $[\mathbf{2 2}, \mathbf{2 5}]$. We claim that $\sigma\left(T^{\prime}\right)=\overline{\mathbb{D}}$ and $\sigma_{\mathrm{ap}}\left(T^{\prime}\right)=\partial \mathbb{D}$.

Let $\mu \in \sigma_{\text {ap }}\left(T^{\prime}\right)$. By Theorem 2.9, the operator $T^{\prime}$ is a hyponormal contraction. Hence, by a result due to Bunce [10, Proposition 12.1, Chapter II], there exists a non-zero *-homomorphism $\phi$ on $C^{*}\left(T^{\prime}\right)$ such that $\phi\left(T^{\prime}\right)=\mu$. By Lemma 2.13, $C^{*}\left(T^{\prime}\right)=C^{*}(T)$. Since $\phi\left(T^{\prime}\right) \neq 0$ and $T T^{* *} T^{\prime}=T^{\prime}$, one has $\phi(T) \overline{\phi\left(T^{\prime}\right)}=1$. Therefore, $\phi$ is a non-zero *-homomorphism on $C^{*}(T)$ such that $\phi(T)=\bar{\mu}^{-1}$. It is easy to see that if $S$ is a bounded linear operator on $\mathcal{H}$, then $\phi(S) \in \sigma_{\text {ap }}(S)$ for every non-zero $*$-homomorphism $\phi$ on $C^{*}(S)$. Consequently, $\bar{\mu}^{-1} \in \sigma_{\text {ap }}(T)=\partial \mathbb{D}$ and hence $\sigma_{\text {ap }}\left(T^{\prime}\right) \subset \partial \mathbb{D}$. Since the boundary of the spectrum is always contained in the approximate point spectrum and since $0 \in \sigma\left(T^{\prime}\right)$, $\sigma_{\text {ap }}\left(T^{\prime}\right) \subset \partial \mathbb{D}$, one has $\sigma\left(T^{\prime}\right)=\overline{\mathbb{D}}$. Therefore, $\sigma_{\text {ap }}\left(T^{\prime}\right)=\partial \mathbb{D}$. This completes the proof of the claim as well as the lemma.

The following proposition says that the non-abelian $C^{*}$-algebra $C^{*}(T)$ generated by a pure 2-hyperexpansive $T$ in $\mathcal{B}(\mathcal{H})$ possesses quite a rich supply of non-zero $*$-homomorphisms.

Proposition 2.15. If $T$ is a pure 2-hyperexpansion, then for every $\mu \in \partial \mathbb{D}$ there exists a non-zero $*$-homomorphism $\phi_{\mu}$ on the $C^{*}$-algebra $C^{*}(T)$ generated by $T$ such that $\phi_{\mu}(T)=\mu$.

Proof. Suppose that $T$ is a pure 2-hyperexpansion. Then $T$ is not invertible [25]. Hence, by Lemma $2.14 \sigma_{\text {ap }}\left(T^{\prime}\right)=\partial \mathbb{D}$. An examination of the proof of this lemma gives the following: for every $\mu \in \sigma_{\text {ap }}\left(T^{\prime}\right)$, there exists a non-zero $*$-homomorphism $\phi_{\mu}$ on the $C^{*}$-algebra $C^{*}(T)$ generated by $T$ such that $\phi_{\mu}(T)=\bar{\mu}^{-1}$. The result now follows.

Let $\mathcal{J}_{S}$ denote the commutator ideal of $C^{*}(S)$, that is, $\mathcal{J}_{S}$ is the norm closed ideal of $C^{*}(S)$ generated by the set of all elements of $C^{*}(S)$ of the form $A B-B A, A, B \in C^{*}(S)$. Let $C\left(\sigma_{\text {ap }}(S)\right)$ denote the $C^{*}$-algebra of continuous functions on the approximate point spectrum $\sigma_{\text {ap }}(S)$ of $S$.

Proposition 2.16. Let $T$ in $\mathcal{B}(\mathcal{H})$ be a 2-hyperexpansion. Let $C^{*}(T)$ be the $C^{*}$ algebra generated by $T$ and let $\mathcal{J}_{T}$ be the commutator ideal of $C^{*}(T)$. There then exists an isometric *-isomorphism $\phi: C^{*}(T) / \mathcal{J}_{T} \rightarrow C\left(\sigma_{\text {ap }}(T)\right)$ such that $\phi\left(T+\mathcal{J}_{T}\right)=\cdot$, where - is the identity function $\cdot(z)=z, z \in \sigma_{\mathrm{ap}}(T)$. 
Proof. Let $T$ in $\mathcal{B}(\mathcal{H})$ be a 2-hyperexpansion and let $T^{\prime}$ be the operator Cauchy dual to $T$. By Theorem 2.9, the Cauchy dual operator $T^{\prime}$ is hyponormal. Hence, by $[\mathbf{1 0}$, Corollary 12.4, Chapter II], there exists an isometric $*$-isomorphism $\phi: C^{*}\left(T^{\prime}\right) / \mathcal{J}_{T^{\prime}} \rightarrow$ $C\left(\sigma_{\text {ap }}\left(T^{\prime}\right)\right)$ such that $\phi\left(T^{\prime}+\mathcal{J}_{T^{\prime}}\right)=\cdot$, where $\cdot(z)=z, z \in \sigma_{\text {ap }}(T)$, is the identity function. Now the desired conclusion follows from the preceding lemmas.

An operator $S$ in $\mathcal{B}(\mathcal{H})$ is analytic if $\bigcap_{n \geqslant 0} S^{n} \mathcal{H}=\{0\}$. Every weighted shift operator is analytic $[\mathbf{2 3}]$.

Proposition 2.17. Let $T$ in $\mathcal{B}(\mathcal{H})$ be an analytic left-invertible operator satisfying $T^{*}\left[T^{*}, T\right] T \leqslant 0$. If $\sigma_{\text {ap }}(T) \subset \partial \mathbb{D}$, then $\mathcal{H}=\bigvee_{n \geqslant 0} T^{n}\left(\operatorname{null}\left(T^{*}\right)\right)$.

Proof. We use the technique developed by Shimorin [24] in his work on the Beurlingtype theorem for invariant subspaces of the Bergman shift (see also [12, Chapter 6]). Let $T$ in $\mathcal{B}(\mathcal{H})$ be an analytic left-invertible operator such that $T^{*}\left[T^{*}, T\right] T \leqslant 0$ and $\sigma_{\text {ap }}(T) \subset \partial \mathbb{D}$. By Remark 2.10, the Cauchy dual operator $T^{\prime}$ is a hyponormal contraction. Using $\sigma_{\text {ap }}(T) \subset \partial \mathbb{D}$, and imitating the proof of Lemma 2.14 (see the second paragraph thereof), it can easily be seen that $\sigma_{\text {ap }}\left(T^{\prime}\right) \subset \partial \mathbb{D}$.

Set $\mathcal{H}_{u}^{\prime}=\bigcap_{n \geqslant 0} T^{\prime n} \mathcal{H}$. It is easy to check that $\mathcal{H}_{u}^{\prime}$ is invariant under $T^{\prime}$ and $T^{\prime} \mathcal{H}_{u}^{\prime}=$ $\mathcal{H}_{u}^{\prime}$. Since $\left.T^{\prime}\right|_{\mathcal{H}_{u}^{\prime}}$ is bounded below, $T^{\prime} \mathcal{H}_{u}^{\prime}=\mathcal{H}_{u}^{\prime}$ implies that $\left.T^{\prime}\right|_{\mathcal{H}_{u}^{\prime}}$ is invertible. Since $\sigma_{\text {ap }}\left(\left.T^{\prime}\right|_{\mathcal{H}_{u}^{\prime}}\right) \subset \sigma_{\text {ap }}\left(T^{\prime}\right)$, the preceding discussion leads to $\sigma_{\text {ap }}\left(\left.T^{\prime}\right|_{\mathcal{H}_{u}^{\prime}}\right) \subset \partial \mathbb{D}$. Invertibility of $\left.T^{\prime}\right|_{\mathcal{H}_{u}^{\prime}}$, together with $\sigma_{\text {ap }}\left(\left.T^{\prime}\right|_{\mathcal{H}_{u}^{\prime}}\right) \subset \partial \mathbb{D}$, forces $\sigma\left(\left.T^{\prime}\right|_{\mathcal{H}_{u}^{\prime}}\right) \subset \partial \mathbb{D}$. Also, the restriction of a hyponormal operator to an invariant subspace is hyponormal [10]. Thus, $\left.T^{\prime}\right|_{\mathcal{H}_{u}^{\prime}}$ is a hyponormal operator such that $\sigma\left(\left.T^{\prime}\right|_{\mathcal{H}_{u}^{\prime}}\right) \subset \partial \mathbb{D}$. By Putnam's inequality [10, Theorem 3.1, Chapter IV], $\left.T^{\prime}\right|_{\mathcal{H}_{u}^{\prime}}$ is normal and hence unitary. Hence, it follows that $P_{\mathcal{H}_{u}^{\prime}} T^{*} T^{\prime} h=$ $h$ for any $h \in \mathcal{H}_{u}^{\prime}$, where $P_{\mathcal{H}_{u}^{\prime}}$ is the orthogonal projection from $\mathcal{H}$ onto $\mathcal{H}_{u}^{\prime}$. Therefore, for any $h \in \mathcal{H}_{u}^{\prime},\left\langle h-T^{*} T^{\prime} h, h\right\rangle_{\mathcal{H}}=\left\langle h-P_{\mathcal{H}_{u}^{\prime}} T^{*} T^{\prime} h, h\right\rangle_{\mathcal{H}_{u}^{\prime}}=0$. Since $T^{\prime}$ is a contraction, we must have $\left\|\left(I-T^{* *} T^{\prime}\right)^{1 / 2} h\right\|_{\mathcal{H}}=0$ for any $h \in \mathcal{H}_{u}^{\prime}$. Hence, $T^{* *} T^{\prime} h=h$ for any $h \in \mathcal{H}_{u}^{\prime}$. This observation, together with $T^{\prime} \mathcal{H}_{u}^{\prime}=\mathcal{H}_{u}^{\prime}$, leads to $T^{* *} \mathcal{H}_{u}^{\prime}=\mathcal{H}_{u}^{\prime}$. The rest of the proof is identical to that of $[\mathbf{1 2}$, Theorem 6.14].

The preceding proposition enables us to present an alternative proof of [22, Theorem 1].

Corollary 2.18 (Richter [22, Theorem 1]). Let $T$ in $\mathcal{B}(\mathcal{H})$ be an analytic operator such that $I-2 T^{*} T+T^{* 2} T^{2} \leqslant 0$. If $\mathcal{M}$ is an invariant subspace of $T$, then $\mathcal{M}=\bigvee_{n \geqslant 0} T^{n}\left(\mathcal{M} \cap(T \mathcal{M})^{\perp}\right)$.

Proof. Let $T$ in $\mathcal{B}(\mathcal{H})$ be such that $\bigcap_{n \geqslant 0} T^{n} \mathcal{H}=\{0\}$ and $I-2 T^{*} T+T^{* 2} T^{2} \leqslant 0$. By [22, Lemma 1], $T$ is expansive. Hence, $T$ is an analytic 2-hyperexpansion. Since $\bigcap_{n \geqslant 0} T^{n} \mathcal{M}=\{0\}$ for any invariant subspace $\mathcal{M}$ of $T$ and since restriction of a 2-hyperexpansion to an invariant subspace is again 2-hyperexpansive, it suffices to treat the case in which $\mathcal{M}=\mathcal{H}$. Since a 2-hyperexpansive $T$ satisfies $T^{*}\left[T^{*}, T\right] T \leqslant 0$ (see the proof of Theorem 2.9) and $\sigma_{\text {ap }}(T) \subset \partial \mathbb{D}$ (see Lemma 2.14(i)), the desired conclusion follows from Proposition 2.17. 
We mention that essentially all the results derived in the remainder of the paper are known in the special case of weighted shift operators. Recall that an operator $S$ in $\mathcal{B}(\mathcal{H})$ is trace class if and only if $\sum_{n \geqslant 0}\left\langle\left(S^{*} S\right)^{1 / 2} e_{n}, e_{n}\right\rangle$ is finite for every choice of orthonormal basis $\left\{e_{n}\right\}_{n \geqslant 0}$. The number $\|S\|_{1} \equiv \sum_{n \geqslant 0}\left\langle\left(S^{*} S\right)^{1 / 2} e_{n}, e_{n}\right\rangle$ is independent of the choice of orthonormal basis and is called the trace norm of $S$. In the case when $S$ is a trace class operator, the trace of $S$ given by $\operatorname{tr}(S)=\sum_{n \geqslant 0}\left\langle S e_{n}, e_{n}\right\rangle$ is independent of the choice of orthonormal basis and is finite. Recall that every trace class operator is compact [10].

An operator $S$ in $\mathcal{B}(\mathcal{H})$ is said to be finitely multicyclic if there are a finite number of vectors $h_{1}, \ldots, h_{m}$ in $\mathcal{H}$ such that

$$
\mathcal{H}=\vee\left\{S^{k} h_{1}, \ldots, S^{k} h_{m}: k \geqslant 0\right\} .
$$

For a positive integer $m, S$ is said to be $m$-multicyclic if it satisfies (2.4) for some set $\left\{h_{1}, \ldots, h_{m}\right\}$ of $m$ vectors in $\mathcal{H}$ and no set of $m-1$ vectors has property (2.4). $S$ is cyclic if it is 1 -cyclic. In that case, there exists a vector $e_{0} \in \mathcal{H}$ such that $\mathcal{H}=\vee\left\{S^{k} e_{0}: k \geqslant 0\right\}$. The vector $e_{0}$ is referred to as a cyclic vector for $S$. For the basic properties of finitely multicyclic operators, the reader is referred to [13].

Since there are 2-hyperexpansive operators with trace class self-commutators (e.g. the Dirichlet shift), and since the only hyponormal 2-hyperexpansions are isometries (justification: if $T$ is a hyponormal 2-hyperexpansion, then $\|T\|=$ the spectral radius of $T[\mathbf{1 0}]$ and the spectral radius of $T$ is equal to 1 (Lemma 2.14 (i)), forcing $T$ to be an isometry), a version of Berger-Shaw theorem for 2-hyperexpansions is desirable. First, a lemma.

Lemma 2.19. Let $T$ in $\mathcal{B}(\mathcal{H})$ be an analytic 2-hyperexpansion and let $T^{\prime}$ be the operator Cauchy dual to $T$. The following statements are then equivalent:

(i) $T$ is finitely multicyclic;

(ii) $T^{\prime}$ is finitely multicyclic;

(iii) $\operatorname{null}\left(T^{*}\right)$ is finite dimensional.

If $T$ satisfies one of the preceding conditions, then the following statements hold:

(a) $\sigma_{e}(T)=\sigma_{\mathrm{e}}\left(T^{\prime}\right)=\partial \mathbb{D}$, and

(b) index $(T-\mu I)=\operatorname{index}\left(T^{\prime}-\mu I\right)=-m(T)=-m\left(T^{\prime}\right)$ for every $\mu \in \mathbb{D}$, where $m(T)$ and $m\left(T^{\prime}\right)$ denote multiplicities of $T$ and $T^{\prime}$, respectively.

Proof. Let $S$ be an $m$-multicyclic operator for some positive integer $m$. By [13, Proposition 1 (i)], nullity $\left(S^{*}-\mu I\right) \leqslant m$ for every $\mu \in \mathbb{C}$. Hence, (i) $\Rightarrow$ (iii) and (ii) $\Rightarrow$ (iii) follow.

Let $T$ in $\mathcal{B}(\mathcal{H})$ be an analytic 2-hyperexpansion and let $T^{\prime}$ be the Cauchy dual operator. By Richter's theorem (Corollary 2.18), one has $\mathcal{H}=\bigvee_{n \geqslant 0} T^{n}\left(\operatorname{null}\left(T^{*}\right)\right.$ ). Thus, (iii) $\Rightarrow$ (i) holds. Also, by [24, Proposition 2.7 (ii)], one has

$$
\left(\bigcap_{n \geqslant 0} T^{n} \mathcal{H}\right)^{\perp}=\bigvee_{n \geqslant 0} T^{\prime n}\left(\operatorname{null}\left(T^{\prime *}\right)\right) .
$$


Since $T$ is analytic and $\operatorname{null}\left(T^{\prime *}\right)=\operatorname{null}\left(T^{*}\right)$, it follows that $\mathcal{H}=\bigvee_{n \geqslant 0} T^{\prime n}\left(\operatorname{null}\left(T^{*}\right)\right)$. The implication (iii) $\Rightarrow$ (ii) is now obvious.

Now suppose that $T$ is an analytic finitely multicyclic 2-hyperexpansion with multiplicity $m(T)$ and that $T^{\prime}$ is the Cauchy dual operator with multiplicity $m\left(T^{\prime}\right)$. Since $T$ is analytic, $T$ is not invertible. Hence, by Lemma 2.14 (ii), $T-\mu I$ and $T^{\prime}-\mu I$ are left semi-Fredholm operators for every $\mu \in \mathbb{D}$. Also, since nullity $\left(T^{*}-\mu I\right)<\infty$ and nullity $\left(T^{*}-\mu I\right)<\infty$ for every $\mu \in \mathbb{D}$ (see the first paragraph of the proof), we must have $\sigma_{\mathrm{e}}(T) \cap \mathbb{D}=\sigma_{\mathrm{e}}\left(T^{\prime}\right) \cap \mathbb{D}=\emptyset$. It is recorded in [25] that $\partial \mathbb{D} \subset \sigma_{\mathrm{e}}(T)$. Since $\sigma\left(T^{\prime}\right)=\overline{\mathbb{D}}$ (Lemma 2.14(ii)), it follows from the elementary Fredholm theory that $\partial \mathbb{D} \subset \sigma_{\mathrm{e}}\left(T^{\prime}\right)$ (see, for example, [11, Theorem 6.8, Chapter XI $]$ ). Hence, $\sigma_{\mathrm{e}}(T)=$ $\sigma_{\mathrm{e}}\left(T^{\prime}\right)=\partial \mathbb{D}$ and (a) follows. Using the continuity of the index, it is easy to see that $\operatorname{index}(T-\mu I)=\operatorname{index}\left(T^{\prime}-\mu I\right)=-\operatorname{nullity}\left(T^{*}\right), \mu \in \mathbb{D}$. Finally, by [13, Theorem 1 (i)], one has nullity $\left(T^{*}\right)=m\left(T^{\prime}\right)=m(T)$ and (b) follows. This completes the proof of the lemma.

Remark 2.20. Note that if $T$ is an analytic 2-hyperexpansion, then $T$ is Fredholm if and only if $T-\mu I$ is Fredholm for every $\mu$ in the unit disc $\mathbb{D}$. Also, we remark that the equivalence of (i), (ii) and (iii) in the preceding lemma holds for any analytic leftinvertible $T$ in $\mathcal{B}(\mathcal{H})$ satisfying $T^{*}\left[T^{*}, T\right] T \leqslant 0$ and $\sigma_{\text {ap }}(T) \subset \partial \mathbb{D}$ (see Remark 2.10 and Proposition 2.16).

It is recorded in [4] that the self-commutator of a completely hyperexpansive weighted shift operator is trace class. Here is a substantial refinement.

Proposition 2.21. Let $T$ in $\mathcal{B}(\mathcal{H})$ be an $m$-multicyclic 2-hyperexpansion and let $T^{\prime}$ be the operator Cauchy dual to $T$. Then $T$ and $T^{\prime}$ have trace class self-commutators.

If in addition $T$ is analytic, then

$$
\operatorname{tr}\left[p\left(T^{\prime}, T^{*}\right), q\left(T^{\prime}, T^{* *}\right)\right]=\operatorname{tr}\left[p\left(T, T^{*}\right), q\left(T, T^{*}\right)\right]=\frac{m}{\pi} \int_{\mathbb{D}}\left(\frac{\partial p}{\partial \bar{z}} \frac{\partial q}{\partial z}-\frac{\partial p}{\partial z} \frac{\partial q}{\partial \bar{z}}\right)(z, \bar{z}) \mathrm{d} A(z)
$$

for all polynomials $p, q$ in two complex variables.

Proof. Suppose that $T$ is an $m$-multicyclic 2-hyperexpansion. By the Wold-type decomposition theorem for 2-hyperexpansions [24, Theorem 3.6], one has

$$
T=U \oplus A \quad \text { on } \mathcal{H}=\mathcal{H}_{u} \oplus \mathcal{H}_{a},
$$

where $U$ is unitary on $\mathcal{H}_{u}$ and $A$ is an analytic 2-hyperexpansion on $\mathcal{H}_{a}$. Since $\left[T^{*}, T\right]=$ $0 \oplus\left[A^{*}, A\right]$, it suffices to check that $\left[A^{*}, A\right]$ is a trace class operator. Since $T$ is finitely multicyclic, $\operatorname{null}\left(T^{*}\right)$ is finite dimensional. Since $\operatorname{null}\left(A^{*}\right) \subset \operatorname{null}\left(T^{*}\right)$ and $A$ is analytic, by the previous lemma $A^{\prime}$ is finitely multicyclic. Also, by Theorem $2.9, A^{\prime}$ is hyponormal. Thus, $A^{\prime}$ is a finitely multicyclic hyponormal operator. By the Berger-Shaw theorem $[\mathbf{1 0}$, Theorem 2.1], we must have $\left\|\left[A^{\prime *}, A^{\prime}\right]\right\|_{1}<\infty$, where $\|\cdot\|_{1}$ denotes the trace norm. It is easy to check that

$$
\left[A^{*}, A\right] A=-A^{*} A\left(\left[A^{\prime *}, A^{\prime}\right] A\right) A^{*} A .
$$


Since $\|X Y\|_{1} \leqslant\|X\|\|Y\|_{1}$ and $\|Y X\|_{1} \leqslant\|Y\|_{1}\|X\|$ for a bounded linear operator $X$ and a trace class operator $Y$, the operator $\left[A^{*}, A\right] A$ is trace class. Hence, $\left[A^{*}, A\right] A A^{\prime *}$ is a trace class operator. But $A A^{\prime *}=I-P_{\operatorname{null}\left(A^{*}\right)}$ and $P_{\operatorname{null}\left(A^{*}\right)}$ is a finite-rank operator. Thus,

$$
\left[A^{*}, A\right] A A^{*}=\left[A^{*}, A\right]\left(I-P_{\operatorname{null}\left(A^{*}\right)}\right)=\left[A^{*}, A\right]-\left[A^{*}, A\right] P_{\operatorname{null}\left(A^{*}\right)} .
$$

Consequently, $\left[A^{*}, A\right]$ is a finite-rank perturbation of the trace class operator $\left[A^{*}, A\right] A A^{\prime *}$. Hence, $\left[A^{*}, A\right]$ is a trace class operator as well.

Let $T^{\prime}$ be the operator Cauchy dual to $T$. Using the decomposition (2.5) of $T$, it is easy to see that

$$
T^{\prime}=U \oplus A^{\prime} \quad \text { on } \mathcal{H}=\mathcal{H}_{u} \oplus \mathcal{H}_{a} .
$$

Since $A^{\prime}$ has a trace class self-commutator (as observed in the preceding paragraph) and $U$ is unitary, $T^{\prime}$ has a trace class self-commutator.

To prove the remaining part, assume in addition that $T$ is analytic and let $p$ and $q$ be any polynomials in two complex variables. Since $T$ has a trace class self-commutator, by a result due to Carey et al. [20, Theorem 2.4] one has

$$
\operatorname{tr}\left[p\left(T, T^{*}\right), q\left(T, T^{*}\right)\right]=\frac{1}{\pi} \int_{\sigma(T)}\left\{\left(\frac{\partial p}{\partial \bar{z}} \frac{\partial q}{\partial z}-\frac{\partial p}{\partial z} \frac{\partial q}{\partial \bar{z}}\right)(z, \bar{z})\right\} \cdot G_{T}(z) \mathrm{d} A(z)
$$

for all polynomials $p, q$ in two complex variables, where $G_{T}$ stands for the Pincus principal function and $\mathrm{d} A$ denotes the Lebesgue planar measure. Since $T$ is a non-invertible 2hyperexpansion, $\sigma(T)=\overline{\mathbb{D}}$ (Lemma 2.14). Also, since $\sigma_{\mathrm{e}}(T)=\partial \mathbb{D}$, index $(T-z I)=-m$, $z \in \mathbb{D}$ (Lemma 2.19) and since $G_{T}(z)=-\operatorname{index}(T-z I)$ for every $z \notin \sigma_{\mathrm{e}}(T)[\mathbf{2 1}$, p. 540] one has

$$
\operatorname{tr}\left[p\left(T, T^{*}\right), q\left(T, T^{*}\right)\right]=\frac{m}{\pi} \int_{\mathbb{D}}\left(\frac{\partial p}{\partial \bar{z}} \frac{\partial q}{\partial z}-\frac{\partial p}{\partial z} \frac{\partial q}{\partial \bar{z}}\right)(z, \bar{z}) \mathrm{d} A(z) .
$$

Also, since $T^{\prime}$ has a trace class self-commutator, using Lemmas 2.14, 2.19 and arguing similarly, one can see that

$$
\operatorname{tr}\left[p\left(T^{\prime}, T^{*}\right), q\left(T^{\prime}, T^{* *}\right)\right]=\frac{m}{\pi} \int_{\mathbb{D}}\left(\frac{\partial p}{\partial \bar{z}} \frac{\partial q}{\partial z}-\frac{\partial p}{\partial z} \frac{\partial q}{\partial \bar{z}}\right)(z, \bar{z}) \mathrm{d} A(z)
$$

for all polynomials $p, q$ in two complex variables. This completes the proof of the proposition.

Example 2.22. Let $\mathbb{D}$ be the open unit disc $\{z \in \mathbb{C}:|z|<1\}$ of the complex plane $\mathbb{C}$ and let $\mathbb{R}^{+}$be the set of positive reals. Let $L^{2}(\mathbb{D}, w)$ denote the Lebesgue space of measurable square-integrable functions with respect to the weighted area measure $w \mathrm{~d} A$ with an area summable weight function $w: \overline{\mathbb{D}} \rightarrow \mathbb{R}^{+}$. Let $L_{a}^{2}(\mathbb{D}, w)$ be the weighted Bergman space $\left\{f \in L^{2}(\mathbb{D}, w): f\right.$ is analytic on $\left.\mathbb{D}\right\}$. Suppose that $1 / w: \overline{\mathbb{D}} \rightarrow \mathbb{R}^{+}$is continuous. Arguing as in the unweighted case, it can be checked that $L_{a}^{2}(\mathbb{D}, w)$ is a Hilbert space $[\mathbf{1 0}, \S 8$, Chapter II]. Let $S$ be the Bergman operator, that is the operator of multiplication by the coordinate function $\cdot$ on $L_{a}^{2}(\mathbb{D}, w)$. The following proposition (essentially due to Shimorin) provides a partial converse to Theorem 2.9; we refer the reader to $[\mathbf{6}]$ for the partial converse of Proposition 2.6. 
Proposition 2.23. Let $S$ be the Bergman operator on $L_{a}^{2}(\mathbb{D}, w)$. If the weight function $w$ is such that $\log w$ is subharmonic, then $S$ is left-invertible and the operator Cauchy dual to $S$ is 2-hyperexpansive.

Proof. The desired conclusions follow from [12, Proposition 9.3] and the remark in the last paragraph of $[\mathbf{2 4}$, p. 168] (see also the discussion following [24, Corollary 4.2]).

Choose the weight function $w$ such that

(1) $\log w$ is subharmonic, and

(2) polynomials are dense in $L_{a}^{2}(\mathbb{D}, w)$.

By (1) and Proposition 2.23, $S^{\prime}$ is 2-hyperexpansive. By (2), the multiplication operator $S$ on $L_{a}^{2}(\mathbb{D}, w)$ is cyclic with cyclic vector 1 . Additionally, it can be easily derived from $[\mathbf{2 4}$, Proposition 2.7] that the Cauchy dual operator $S^{\prime}$ is analytic. Hence, by Lemma 2.19, the Cauchy dual operator $S^{\prime}$ is cyclic. Now it follows from Proposition 2.21 that $S$ and $S^{\prime}$ have trace class self-commutators, and that

$$
\operatorname{tr}\left[p\left(S, S^{*}\right), q\left(S, S^{*}\right)\right]=\operatorname{tr}\left[p\left(S^{\prime}, S^{\prime *}\right), q\left(S^{\prime}, S^{\prime *}\right)\right]=\frac{1}{\pi} \int_{\mathbb{D}}\left(\frac{\partial p}{\partial \bar{z}} \frac{\partial q}{\partial z}-\frac{\partial p}{\partial z} \frac{\partial q}{\partial \bar{z}}\right)(z, \bar{z}) \mathrm{d} A(z)
$$

for all polynomials $p$ and $q$ in two complex variables.

Corollary 2.24. If $T$ in $\mathcal{B}(\mathcal{H})$ is an m-multicyclic 2-hyperexpansion and $T^{\prime}$ is the Cauchy dual operator, then $T, T^{\prime}$ have trace class self-commutators and

$$
\operatorname{tr}\left[T^{* *}, T^{\prime}\right]=\operatorname{tr}\left[T^{*}, T\right] \leqslant \frac{m}{\pi} \operatorname{area}(\sigma(T))=\frac{m}{\pi} \operatorname{area}\left(\sigma\left(T^{\prime}\right)\right) .
$$

If in addition $T$ is analytic, then

$$
\operatorname{tr}\left[T^{\prime *}, T^{\prime}\right]=\operatorname{tr}\left[T^{*}, T\right]=m
$$

Proof. Let $T$ in $\mathcal{B}(\mathcal{H})$ be an $m$-multicyclic 2-hyperexpansion and let $T^{\prime}$ be the Cauchy dual operator. Consider decompositions $(2.5),(2.6)^{\prime}$. If $\mathcal{H}_{a}=\{0\}$, then $T$ is unitary and $T=T^{\prime}$. Hence, equality holds in (2.6). Suppose that $\mathcal{H}_{a} \neq\{0\}$. It is clear from (2.5), (2.6) that $\left[T^{\prime *}, T^{\prime}\right]=0 \oplus\left[A^{\prime *}, A^{\prime}\right],\left[T^{*}, T\right]=0 \oplus\left[A^{*}, A\right]$. Since $A$ is an analytic finitely multicyclic 2-hyperexpansion with multiplicity $m(A)$, by Proposition 2.21 we obtain $\operatorname{tr}\left[A^{\prime *}, A^{\prime}\right]=$ $\operatorname{tr}\left[A^{*}, A\right]=m(A)$. Since $T$ extends $A$, one has $m(A) \leqslant m$. Thus, $\operatorname{tr}\left[T^{*}, T^{\prime}\right]=\operatorname{tr}\left[T^{*}, T\right] \leqslant$ $m$. Since $\sigma\left(T^{\prime}\right)=\sigma(T)=\overline{\mathbb{D}}$ (Lemma 2.14), the inequality in (2.6) follows. This completes the proof of the corollary.

Remark 2.25. For hyponormal operators, one can easily deduce the Putnam inequality from the Berger-Shaw theorem [10]. In view of the preceding corollary, one may expect a version of Putnam's inequality for 2-hyperexpansions.

Surprisingly,

$$
\left\|T^{*} T-T T^{*}\right\| \geqslant \frac{1}{\pi} \operatorname{area}(\sigma(T))
$$


holds true for a 2-hyperexpansive $T$ in $\mathcal{B}(\mathcal{H})$. Moreover, equality holds in (2.7) for a 2-hyperexpansive $T$ if and only if $T$ is an isometry. One may argue as follows. Let $T$ be a 2-hyperexpansion. Consider the decomposition (2.5). If $\mathcal{H}_{a}=\{0\}$, then $T$ is unitary and equality holds in (2.7). Assume that $\mathcal{H}_{a} \neq\{0\}$. Thus, one can choose $h \in \mathcal{H}$ such that $\|h\|=1$ and $A^{*} h=0$. Hence,

$$
\left\|T^{*} T-T T^{*}\right\|=\left\|A^{*} A-A A^{*}\right\| \geqslant\left|\left\langle\left(A^{*} A-A A^{*}\right) h, h\right\rangle\right|=\|A h\|^{2} \geqslant\|h\|^{2}=1 .
$$

Also, $(1 / \pi) \operatorname{area}(\sigma(T))=1$ (Lemma 2.14). Hence, $\left\|T^{*} T-T T^{*}\right\| \geqslant(1 / \pi) \operatorname{area}(\sigma(T))$ follows. To prove the 'moreover' part, suppose that $T$ is an isometry. Then $T$ is hyponormal as well as 2-hyperexpansive. Hence, by Putnam's inequality and (2.7), equality holds in (2.7). Now suppose that equality holds in (2.7) for a 2-hyperexpansive $T$ in $\mathcal{B}(\mathcal{H})$ and that $\mathcal{H}_{a} \neq\{0\}$. Note that equality in (2.7) leads to $\left\|A^{*} A-A A^{*}\right\|=1$. An examination of (2.8) yields $\|A h\|=\|h\|, h \in \operatorname{null}\left(A^{*}\right)$. Using the 2-hyperexpansivity of $A$, it can easily be seen that $\left\|A^{k} h\right\|=\|h\|, h \in \operatorname{null}\left(A^{*}\right), k \geqslant 0$. Since $A$ is expansive, one has $A^{* k} A^{k} h=h$ for every $h \in \operatorname{null}\left(A^{*}\right), k \geqslant 0$. Hence, $A^{m} \operatorname{null}\left(A^{*}\right) \perp A^{n} \operatorname{null}\left(A^{*}\right)$ for every $m, n \geqslant 0$ such that $m \neq n$. Imitating the proof of the von Neumann-Wold-Kolmogorov decomposition theorem for isometries, it can now be seen that $A$ is a unilateral shift of multiplicity nullity $\left(A^{*}\right)[\mathbf{1 0}]$. Hence, $T$ is an isometry.

In the proof of Proposition 2.21, we did not use the full strength of the Berger-Shaw theorem. The general version of the Berger-Shaw theorem says that every rationally cyclic hyponormal has a trace class self-commutator [20]. This and Proposition 2.21 suggest the following conjecture.

Conjecture 2.26. Every rationally cyclic 2-hyperexpansive operator has a trace class self-commutator.

Corollary 2.27. Let $T$ in $\mathcal{B}(\mathcal{H})$ be a finitely multicyclic analytic irreducible 2-hyperexpansive operator and let $C^{*}(T)$ be the $C^{*}$-algebra generated by $T$. If $\mathcal{C}(\mathcal{H})$ denotes the algebra of compact operators on $\mathcal{H}$, then we have an exact sequence of $C^{*}$-algebras

$$
0 \mapsto \mathcal{C}(\mathcal{H}) \stackrel{i}{\hookrightarrow} C^{*}(T) \stackrel{\pi}{\mapsto} C(\partial \mathbb{D}) \mapsto 0,
$$

where $i: \mathcal{C}(\mathcal{H}) \hookrightarrow C^{*}(T)$ is the inclusion map and $\pi: C^{*}(T) \rightarrow C(\partial \mathbb{D})$ is the unital $*$-homomorphism defined by $\pi(T)=\cdot$, where $\cdot$ is the identity function $\cdot(z)=z, z \in \partial \mathbb{D}$.

Proof. Let $T$ in $\mathcal{B}(\mathcal{H})$ be a finitely multicyclic analytic irreducible 2-hyperexpansion. By Corollary 2.24, the self-commutator of $T$ is compact. It now follows from $[\mathbf{1 0}$, Lemma 12.9 , Chapter II] that the commutator ideal of $T$ is $\mathcal{C}(\mathcal{H})$, the algebra of compact operators on $\mathcal{H}$. Since $0 \in \sigma(T)$, by Lemma 2.14 (ii), $\sigma_{\text {ap }}(T)$ is equal to the unit circle $\partial \mathbb{D}$. The desired conclusion now follows from Proposition 2.16.

Corollary 2.28. If $T$ in $\mathcal{B}(\mathcal{H})$ is an m-multicyclic 2-hyperexpansion, then

$$
T=T_{0} \oplus T_{1} \oplus \cdots \quad \text { on } \mathcal{H}=\mathcal{H}_{0} \oplus \mathcal{H}_{1} \oplus \cdots,
$$

where $T_{0}$ is unitary and, for $n \geqslant 1, T_{n}$ is an $m_{n}$-multicyclic irreducible 2-hyperexpansion operator on the infinite-dimensional space $\mathcal{H}_{n}$ for some integer $m_{n} \leqslant m$.

Except for the ordering of the summands, this decomposition is unique. 
Proof. Let $T$ in $\mathcal{B}(\mathcal{H})$ be an $m$-multicyclic 2-hyperexpansion. By Corollary 2.24 the self-commutator of $T$ is compact. Since a normal 2-hyperexpansion is unitary, the required result follows from [10, Theorem 5.4, Chapter IV].

It was pointed out by the referee that an explicit model for cyclic completely hyperexpansive operators is given by Aleman [3]. The following result known in the case of weighted shift operators $[\mathbf{8}, \mathbf{1 7}]$ asserts that an analytic $m$-multicyclic 2 -hyperexpansion is essentially the unilateral shift of multiplicity $m$.

Corollary 2.29. Every analytic finitely multicyclic 2-hyperexpansive $T$ in $\mathcal{B}(\mathcal{H})$ is unitarily equivalent to a compact perturbation of a unilateral shift.

Proof. Suppose that $T$ in $\mathcal{B}(\mathcal{H})$ is an analytic $m$-multicyclic 2-hyperexpansion. By Lemma $2.19, \sigma_{\mathrm{e}}(T)=\partial \mathbb{D}$ and $\operatorname{index}(T-\mu I)=-m, \mu \in \mathbb{D}$. Also, by Corollary 2.24 , the self-commutator of $T$ is compact. Clearly, the unilateral shift $U$ of multiplicity $m$ has compact self-commutator and $U$ satisfies $\sigma_{\mathrm{e}}(U)=\sigma_{\mathrm{e}}(T)$, index $(U-\mu I)=\operatorname{index}(T-\mu I)$, $\mu \in \mathbb{C} \backslash \partial \mathbb{D}[\mathbf{1 0}]$. The desired conclusion now follows from the following result of Brown et al. [9]: if $S_{1}, S_{2} \in \mathcal{B}(\mathcal{H})$ are essentially normal operators, then $S_{1}$ is unitarily equivalent to a compact perturbation of $S_{2}$ if and only if $\sigma_{\mathrm{e}}\left(S_{1}\right)=\sigma_{\mathrm{e}}\left(S_{2}\right)$, and $\operatorname{index}\left(S_{1}-\mu I\right)=$ $\operatorname{index}\left(S_{2}-\mu I\right)$ for every $\mu \in \mathbb{C} \backslash \sigma_{\mathrm{e}}\left(S_{1}\right)$.

Remark 2.30. Let $T$ in $\mathcal{B}(\mathcal{H})$ be an $m$-multicyclic 2-hyperexpansion. It follows from (2.5) and Corollary 2.29 that $\Delta_{T} \equiv T^{*} T-I$ and $\Delta_{T^{*}} \equiv T T^{*}-I$ are compact. This generalizes [2, Proposition 1.24]. If, in addition, $T$ is analytic, then it is clear from Corollary 2.29 that the Weyl spectrum $\sigma_{w}(T)$ of $T$ is equal to the Weyl spectrum $\sigma_{w}(U)$ of a unilateral shift $U$ of multiplicity $m$. Since $\sigma_{w}(U)=\overline{\mathbb{D}}[\mathbf{1 0}]$, one has $\sigma_{w}(T)=\overline{\mathbb{D}}$.

A necessary condition for an operator $T$ in $\mathcal{B}(\mathcal{H})$ to be unitarily equivalent to a compact perturbation of a unilateral shift $U$ is that the essential spectrum of $T$ is equal to the essential spectrum of $U$. Also, if the multiplicity of $U$ is equal to nullity $(T)$ for an analytic 2-hyperexpansive $T$, then, by the continuity of index, one has $\sigma_{\mathrm{e}}(U)=\sigma_{\mathrm{e}}(T)$. Hence it is interesting to consider the following question.

Question 2.31. Is every analytic 2-hyperexpansive operator unitarily equivalent to a compact perturbation of a unilateral shift?

Acknowledgements. A substantial part of this paper was written while the author visited the Indian Institute of Technology, Bombay: the author thanks the faculty, the research scholars and the administrative unit of the Department of Mathematics for their warm hospitality. In particular the author thanks Professor A. Athavale for his constant guidance and encouragement throughout the preparation of this paper.

\section{References}

1. J. Agler, Hypercontractions and subnormality, J. Operat. Theory 13 (1985), 203-217.

2. J. Agler and M. Stankus, $m$-isometric transformations of Hilbert spaces, I, Integ. Eqns Operat. Theory 21 (1995), 383-429. 
3. A. Aleman, The multiplication operators on Hilbert spaces of analytic functions, Habilitationsschrift, FernUniversität in Hagen (1993).

4. A. Athavale, On completely hyperexpansive operators, Proc. Am. Math. Soc. 124 (1996), 3745-3752.

5. A. Athavale, The complete hyperexpansivity analog of the Embry conditions, Studia Math. 154 (2003), 233-242.

6. A. Athavale And A. Ranjekar, Bernstein functions, complete hyperexpansivity and subnormality, I, Integ. Eqns Operat. Theory 43 (2002), 253-263.

7. A. Athavale And A. Ranjekar, Bernstein functions, complete hyperexpansivity and subnormality, II, Integ. Eqns Operat. Theory 44 (2002), 1-9.

8. A. Athavale and V. Sholapurkar, Completely hyperexpansive operator tuples, Positivity 3 (1999), 245-257.

9. L. Brown, R. Douglas And P. Fillmore, Unitary equivalence modulo the compact operators and extensions of $C^{*}$-algebras, Lecture Notes in Mathematics, Volume 345, pp. 58-128 (Springer, 1973).

10. J. CONWAY, The theory of subnormal operators, Mathematical Surveys and Monographs, Volume 36 (American Mathematical Society, Providence, RI, 1991).

11. J. Conway, A course in functional analysis (Springer, 1997).

12. H. Hedenmalm, B. Korenblum and K. Zhu, Theory of Bergman spaces (Springer, 2000).

13. D. Herrero, On multicyclic operators, Integ. Eqns Operat. Theory 1 (1978), 57-102.

14. Z. JABLOŃSKI, Complete hyperexpansivity, subnormality and inverted boundedness conditions, Integ. Eqns Operat. Theory 44 (2002), 316-336.

15. Z. Jabloński, Hyperexpansive composition operators, Math. Proc. Camb. Phil. Soc. 135 (2003), 513-526.

16. Z. JABLOŃSKI, Hyperexpansive operator-valued unilateral weighted shifts, Glasgow Math. J. 46 (2004), 405-416.

17. Z. Jabloński And J. Stochel, Unbounded 2-hyperexpansive operators, Proc. Edinb. Math. Soc. 44 (2001), 613-629.

18. Z. Jabloński, I. Jung And J. Stochel, Backward extensions of hyperexpansive operators, Studia Math. 173 (2006), 233-257.

19. C. KubRusly, An introduction to models and decompositions in operator theory (Birkhäuser, 1997).

20. M. MARTin AND M. PUtinar, Lectures on hyponormal operators, Operator Theory: Advances and Applications, Volume 39 (Birkhäuser, 1989).

21. J. Pincus, Symmetric singular integral operators, Indiana Univ. Math. J. 23 (1973), 537-556.

22. S. Richter, Invariant subspaces of the Dirichlet shift, J. Reine Angew. Math. 386 (1988), 205-220.

23. A. SHIELDS, Weighted shift operators and analytic function theory, in Topics in Operator Theory, Mathematical Surveys and Monographs, Volume 13, pp. 49-128 (American Mathematical Society, Providence, RI, 1974).

24. S. Shimorin, Wold-type decompositions and wandering subspaces for operators close to isometries, J. Reine Angew. Math. 531 (2001), 147-189.

25. V. Sholapurkar and A. Athavale, Completely and alternatingly hyperexpansive operators, J. Operat. Theory 43 (2000), 43-68.

26. B. SzÖKEFAlvi-NAGy AND C. FoIAs, Harmonic analysis of operators on Hilbert space (North-Holand, Amsterdam, 1970). 